\title{
Microaneurysm Turnover in Diabetic Retinopathy Assessed by Automated RetmarkerDR Image Analysis - Potential Role as Biomarker of Response to Ranibizumab Treatment
}

\author{
Simon F. Leicht ${ }^{\mathrm{a}}$ Marcus Kernt ${ }^{\mathrm{a}}$ Aljoscha Neubauer ${ }^{\mathrm{a}}$ Armin Wolf $^{\mathrm{a}}$ \\ Carlos Manta Oliveira $^{b}$ Michael Ulbig ${ }^{a}$ Christos Haritoglou ${ }^{a}$ \\ a Department of Ophthalmology, Ludwig Maximilian University, Munich, Germany; ${ }^{b}$ Critical Health SA, \\ Coimbra, Portugal
}

\section{Key Words}

Ranibizumab · Diabetic retinopathy · Progression .

Microaneurysm turnover $\cdot$ Retmarker $\cdot$ Diabetic macular edema . Optomap $\cdot$ Automated fundus image analysis

\begin{abstract}
Purpose: To evaluate the influence of a ranibizumab treatment on microaneurysm (MA) turnover in diabetic retinopathy. Methods: Sixty-nine eyes were included in this retrospective study. We compared a group of 33 eyes with ranibizumab treatment for diabetic macular edema to 36 eyes with nonproliferative diabetic retinopathy only. Nonmydriatic ultra-widefield scanning laser ophthalmoscopy (Optomap) images were obtained at a mean $4.76 \pm 1.69$ days prior to the first ranibizumab injection (baseline) and again $35.94 \pm 2.44$ days after the third consecutive injection in a 4-week interval. In untreated controls, images were obtained at baseline and $97.81 \pm 3.16$ days thereafter. Images were analyzed using the RetmarkerDR software (Critical Health SA, Coimbra, Portugal), and the turnover of MAs was documented and analyzed. Thereafter, MA turnover was correlated with central retinal thickness (CRT) as assessed by OCT. Results: At baseline, patients in the treatment group had $5.64 \pm 0.75$ MAs. One month after 3 ranibizumab
\end{abstract}

\section{KARGER}

E-Mail karger@karger.com

www.karger.com/oph injections, measured MAs decreased to $4.03 \pm 0.66$. In the untreated control group, the initial number of $3.36 \pm 0.6 \mathrm{MAs}$ remained almost unchanged over 3-4 months ( $2.89 \pm 0.57 \mathrm{MAs}$ ). Dynamic analysis showed that after ranibizumab treatment $3.06 \pm 0.5$ new MAs appeared, while $5.09 \pm 0.79$ disappeared. In the control group, $2.11 \pm 0.4$ new MAs appeared and $2.61 \pm$ 0.48 disappeared. MA turnover was significantly higher with ranibizumab compared to the control group $(8.15 \pm 1.14$ vs. $4.72 \pm 0.81, p<0.001)$. Consistently, CRT decreased from 444 to $330 \mu \mathrm{m}$ in the ranibizumab group, while there was no change in the control group (291 vs. $288 \mu \mathrm{m}$ ). Conclusion: The treatment of macular edema using ranibizumab does not only reduce macular thickness, but also has an impact on the turnover of MAs in diabetic retinopathy. RetmarkerDR analysis showed that more pre-existent MAs disappeared than new MAs developed, and the absolute number of MAs also decreased.

(c) 2014 S. Karger AG, Basel

\section{Introduction}

Diabetic retinopathy is one of the leading causes of blindness in the western world and accounts for most cases of legal blindness in the working age [1]. The incidence
(C) 2014 S. Karger AG, Basel

0030-3755/14/2314-0198\$39.50/0 
of blindness associated with diabetic retinopathy in Germany was reported to be $0.7 / 100,000$ person-years of observation in a recent study [2].

Visual loss in patients with type 2 diabetes is commonly caused by diabetic macular edema [3].

The Early Treatment Diabetic Retinopathy Study (ETDRS) has shown that a significant reduction of visual loss is achieved by focal photocoagulation of macular edema, although laser photocoagulation is not always beneficial [4]. Alternative promising treatment options for center-involving diabetic macular edema are now available following the introduction of vascular endothelial growth factor (VEGF) inhibitors such as bevacizumab and ranibizumab [5-7], with ranibizumab being the only treatment option with a label.

Besides the potential risk of diabetic macular edema, patients with diabetic retinopathy reveal characteristic signs such as microaneurysms (MAs), dot hemorrhages, hard exudates as a sign of vascular permeability and other abnormalities of retinal vessels such as venous beading and intraretinal microvascular abnormalities [8]. Recent studies have emphasized the turnover of MAs assessed by an automated analysis (RetmarkerDR) as a predictive factor for the progression to clinically significant macular edema (CSME) and to identify patients being at risk to develop sight-threatening complications $[9,10]$.

On the other hand, little is known about the turnover of MAs in patients who already developed center-involving diabetic macular edema and are being treated with an anti-VEGF drug such as ranibizumab. One may hypothesize that the pharmacological treatment of centerinvolving diabetic macular edema not only has an impact on central retinal thickness (CRT) as measured by optical coherence tomography (OCT) and visual acuity, but also on the turnover of MAs, a hallmark of diabetic retinopathy.

Therefore, the present study was conducted to compare MA turnover in diabetic eyes with and without CSME and in the eyes with CSME receiving ranibizumab treatment in order to assess whether there is any association between MA turnover and edema resolution as measured by OCT [10].

\section{Methods}

Study Population and Setup

We retrospectively evaluated fundus images from patients in our diabetic retinopathy outpatient clinic. Two groups of pa- tients were established: group 1 including patients with newly formed fovea-involving diabetic macular edema being scheduled for treatment with ranibizumab, and group 2 including patients with nonproliferative diabetic retinopathy as a control. The diagnosis in patients of groups 1 and 2 was established using slitlamp microscope examination with a 78-dpt lens (Volk, Mentor, Ohio, USA); in group 1 we additionally performed OCT and fluorescein angiography if necessary to confirm the presence of macular edema. Patients in the treatment group underwent 3 consecutive injections of ranibizumab at a 4 -week interval.

A total of 69 eyes of 55 patients (33 treated and 36 untreated eyes) were recruited for the study. At each visit a complete bilateral eye examination, including best-corrected visual acuity, slitlamp microscope examination and fundus examination using a 78-dpt lens, as well as measurement of intraocular pressure were performed. In addition, digital ultra-widefield scanning laser ophthalmoscopy (Optomap) images were taken at each visit. In the present study using RetmarkerDR, images $4.76 \pm 1.69$ days prior to the first ranibizumab injection (baseline) and $35.94 \pm 2.44$ days after the third injection were analyzed in the treatment group. In the control group, a baseline image and a follow-up image after $97.81 \pm 3.16$ days were chosen for automated analysis. OCT examinations were performed at baseline and at each follow-up visit in the treatment group and 3-4 months thereafter in the control group. Fellow eyes of patients undergoing treatment with ranibizumab were not allowed to be included in the control group.

\section{Image Selection and Preparation for the RetmarkerDR \\ Analysis}

Only high-quality images centered on the macular area were retrospectively and consecutively selected. The images were cropped (the periphery outside the retinal arcades was removed) prior to the analysis and then analyzed with the RetmarkerDR software as described previously [10].

\section{Retmarker Analysis}

The RetmarkerDR software compares images of the same fundus area at different time points and registers newly formed or disappeared MAs (red dots). For more detailed information, please refer to the work of Haritoglou et al. [10] and Ribeiro et al. [11].

\section{MA Turnover}

For each patient, both in the treatment and control groups, the total number of MAs, the number of newly formed MAs and the number of MAs which had disappeared was documented by comparing the baseline with the last follow-up visit. The MA turnover over the period of review was calculated by adding the absolute value of disappeared and newly formed MAs comparing baseline images and images obtained at the last visit.

\section{Optical Coherence Tomography}

All OCT examinations were performed with a Heidelberg Engineering Spectralis SD-OCT (Heidelberg Engineering, Heidelberg, Germany). An examination consisted of a volume scan of 49 $B$ scans with a distance between B scans of $118 \mu \mathrm{m}$. OCTs had to be correctly centered, and CRT was documented for both, the baseline and the follow-up visits, respectively. 


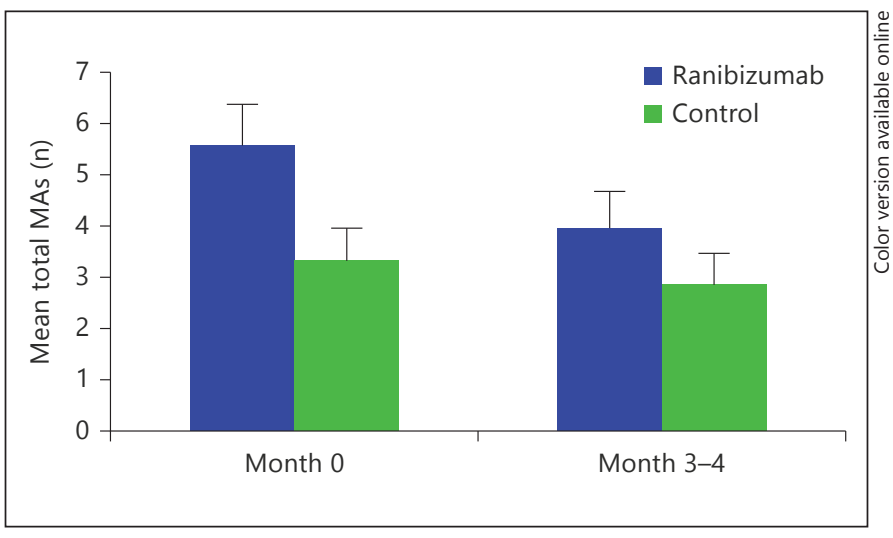

Fig. 1. Mean total MAs in the treated and the control group and their changes over time are shown in absolute numbers. While there was a significant reduction in MA numbers in the treatment group $(\mathrm{p}<0.05)$, there was no change in the control group between baseline and follow-up.

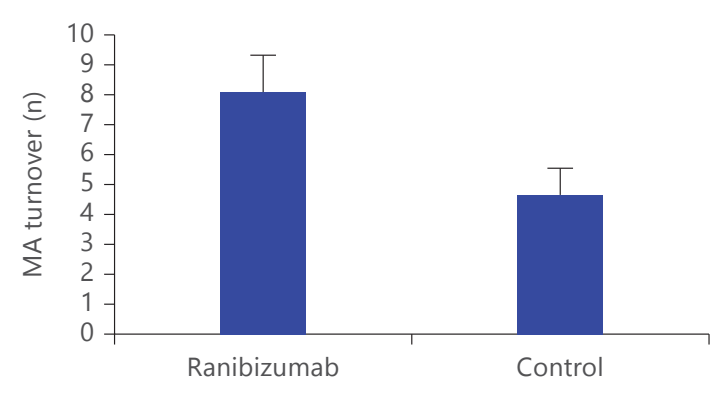

Fig. 2. With $8.15 \pm 1.14$ under ranibizumab treatment, the calculated turnover was significantly higher compared to that in the control group $(4.72 \pm 0.81 ; \mathrm{p}<0.05)$.

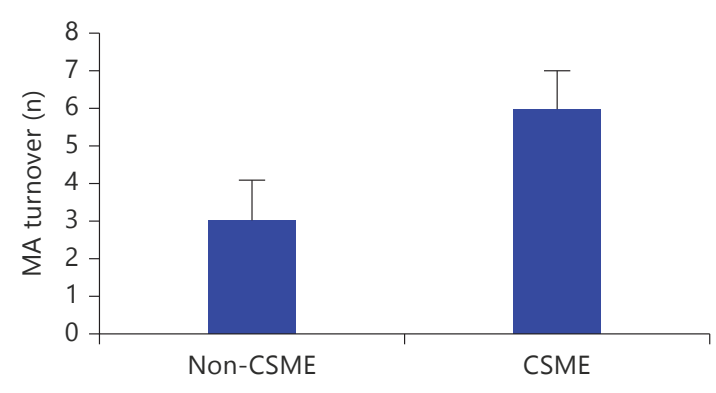

Fig. 3. Here, the MA turnover is shown between baseline and follow-up, given as absolute numbers, for both the non-CSME and the CSME groups $(\mathrm{p}<0.05)$.
Statistical Analysis

All data are shown as means \pm SEM. All results were stored in an Excel work sheet and analyzed with SPSS 21 (IBM Deutschland $\mathrm{GmbH}$, Ehningen, Germany).

\section{Results}

\section{Study Population}

The study population consisted of 31 male and $24 \mathrm{fe}-$ male patients. The mean age was 63.0 years $(\mathrm{SD} \pm 12.1$, range $40-86$ years).

\section{MA Numbers}

Comparing the difference of total numbers of MAs in the treatment and control group, we observed a significant difference at baseline (mean $5.64 \pm 0.75$ vs. $3.36 \pm 0.6 ; \mathrm{p}<0.05$ comparing treatment vs. control; fig. 1) but not at the follow-up visit comparing treated patients with and without CSME (mean $4.03 \pm 0.66$ vs. $2.89 \pm 0.57)$.

Within the treatment group, the mean number of MAs significantly decreased from $5.64 \pm 0.75$ at baseline to $4.03 \pm 0.66$ MAs $1-2$ months after the third ranibizumab injection ( $p<0.05$; fig. 1$)$. In the untreated group, the mean number of $3.36 \pm 0.6$ MAs at baseline remained statistically unchanged until 3-4 months later with $2.89 \pm$ 0.57 MAs being calculated (fig. 1).

In the treatment group, $3.06 \pm 0.5$ new MAs had appeared at the follow-up, while $5.09 \pm 0.79$ had disappeared. In the control group, $2.11 \pm 0.4$ new MAs had appeared at the follow-up, and $2.61 \pm 0.48$ had disappeared. The calculated turnover for both groups differed significantly with $8.15 \pm 1.14$ under ranibizumab treatment and $4.72 \pm 0.81$ in the untreated group (fig. 2 ; p < 0.05).

The comparison of MA turnover values in patients with and without CSME revealed a median turnover of 3 in the group without CSME (CRT $<300 \mu \mathrm{m})$, compared to a value of 6 in the CSME group (CRT $\geq 300 \mu \mathrm{m}$; $\mathrm{p}<$ 0.05 ; fig. 3 ).

\section{OCT Measurements}

At baseline, the mean CRT was $444 \pm 29 \mu \mathrm{m}$ (ranging from 202 to $822 \mu \mathrm{m}$ ) in the treatment group and $291 \pm 22$ $\mu \mathrm{m}$ (ranging from 180 to $723 \mu \mathrm{m}$ ) in the control group $(\mathrm{p}<0.001)$. At the follow-up, CRT had decreased to $330 \pm$ $23 \mu \mathrm{m}$ (ranging from 160 to $604 \mu \mathrm{m}$ ) in the treatment group ( $\mathrm{p}<0.01$ ), while it remained unchanged at $288 \pm$ $18 \mu \mathrm{m}$ in the untreated group (fig. 4). 


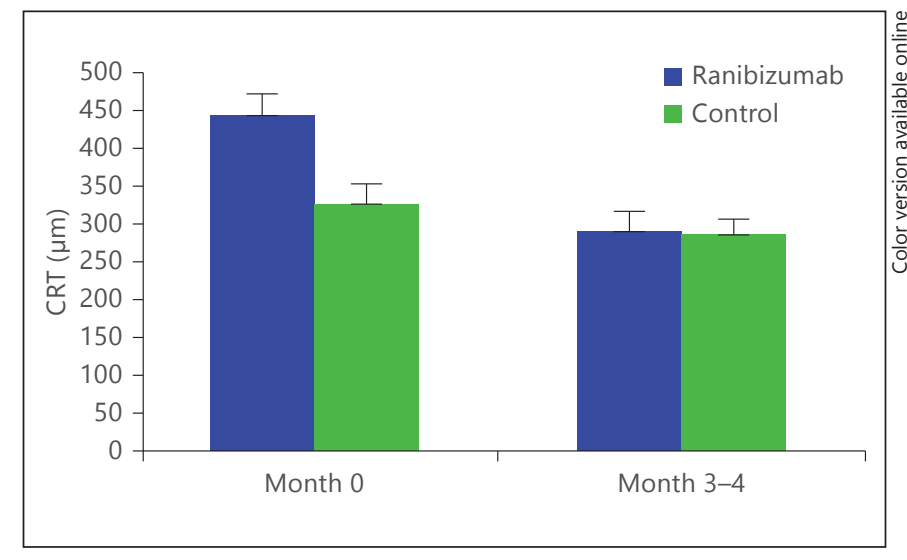

Fig. 4. While the mean CRT significantly differed between both groups at baseline ( $444 \pm 29$ vs. $291 \pm 22 \mu \mathrm{m}$ in controls; $\mathrm{p}<0.001)$, CRT significantly decreased after treatment $(\mathrm{p}<0.01)$ to a similar level as in the control group ( $330 \pm 23$ vs. $288 \pm 18 \mu \mathrm{m}$ in controls).

Looking at the change in CRT and in total MA number between baseline and follow-up, we found a significant correlation $(\mathrm{p}<0.01)$. Moreover, in those cases where a reduction of macular edema was observed, the turnover was significantly higher than in those eyes in which macular edema increased CRT ( $\mathrm{p}<0.05)$.

\section{Discussion}

The presence of MAs, intraretinal hemorrhages and signs of vascular permeability and capillary closure, i.e. hard exudates and cotton wool spots, characterizes nonproliferative diabetic retinopathy.

In general, it is important to monitor the progression of the disease in a specific patient and identify the individual risk of progression to sight-threatening complications such as diabetic macular edema or proliferative diabetic retinopathy. Unfortunately, MAs in diabetic retinopathy do not just accumulate and thus reveal progression of the disease. They appear and disappear as red dots on fundus photographs, and a high turnover is a predictive sign for fast progression to vision-threatening complications such as diabetic macular edema [10].

The evolution and progression of retinal changes are different from patient to patient, making it necessary to closely monitor the individual eye for signs of disease progression. An early detection of those changes allows for timely and adequate treatment and thus for the prevention of irreversible functional loss.

Effect of Ranibizumab on MA Turnover in Diabetic Retinopathy
In 1986, Kohner and Sleightholm [12] first described a correlation between MA counts and the severity of early diabetic retinopathy. Others reported that leaking MAs are associated with retinal thickness in diabetic retinopathy [13]. In recent years, counting numbers of MAs at the posterior pole of the retina and the evaluation of the dynamics in MA turnover, i.e. their appearance and disappearance, have been established as a prognostic factor for worsening of diabetic retinopathy and development of diabetic macular edema $[7,9,11$, 14-17].

While previous investigators had to work with manual methods, like blinded counting by two separate graders [17], an automated software is now available with the RetmarkerDR, providing reproducible analysis without confounders like experience, fatigue etc. and allowing for noninvasive screening, as fundus photography is widely available and easy to use. Due to image coregistration, it automatically provides information about appearance and disappearance of MAs in defined retinal areas, thus providing the formation rate of new MAs compared to an earlier examination.

Not only has there been data about the predictive value of MA turnover for retinopathy progression, but also about MA dynamics under therapy. Two groups reported a reduction of retinal thickness after photocoagulation, correlated with a decrease in numbers of leaking MAs $[18$, 19]. Yet, data about the influence of pharmacological treatment on MAs was missing. Now for the first time we present data for the behavior of MA turnover under ranibizumab treatment.

Ranibizumab has been available for the treatment of diabetic macular edema for a couple of years and has been shown to not only influence visual acuity and CRT, but also to reduce the severity of diabetic retinopathy, according to the modified ETDRS score [20]. Therefore, one may assume that this fact is also reflected in MA turnover. And indeed, in our study we found that the average total number of MAs is decreasing in the ranibizumab group, whereas the number of total MAs remains unchanged over 3-4 months in the untreated group. Also, more MAs vanished in the treated group, resulting in a higher MA turnover in the treatment group when compared to controls.

In previous trials investigating the role of automated fundus analyses in diabetic retinopathy, a higher MA turnover was found to be a predictive factor for the development of diabetic macular edema [10]. Of note this observation was made in patients not undergoing any pharmacological treatment such as anti-VEGF therapy. How- 
ever, in line with this previous report, the MA turnover in the present study was higher in patients with CSME compared to patients without CSME. In addition, the number of MAs was significantly higher in patients with macular edema compared to patients without macular edema.

In the present study, we showed that the intravitreal injection of ranibizumab resulted in a decrease in the number of MAs with an increased turnover of MAs with more MAs disappearing than appearing. In other words, in eyes with diabetic macular edema, anti-VEGF treatment resulted in an attenuation of diabetic fundus changes and an alignment of the fundus changes between eyes undergoing anti-VEGF treatment for diabetic macular edema and eyes without diabetic macular edema. Therefore, one may assume that a high MA turnover not only stands for a higher risk for developing CSME and worsening of diabetic retinopathy in eyes without diabetic macular edema, but also for an increased dynamic of the observed fundus changes in the context of intravitreal pharmacological therapy once macular edema has occurred. This finding might serve as a quantification of the treatment response in the future, and it will be very interesting to investigate potential correlations with other parameters such as a decrease in CRT measured by optical coherence tomography in a larger group of patients. As a preliminary result in this context, we were able to show that the changes in total number of MAs and MA turnover were accompanied by a decrease in macular thickness under ranibizumab treatment in our patients.

Of course one may argue that a comparison of patients with nonproliferative diabetic retinopathy, with and without macular edema, as done in the present study, is critical. The aim of this investigation was to analyze the effect of an anti-VEGF treatment on MA turnover. As a control we chose a group of patients in whom one may not expect fundus changes over the period of review. This approach had the following reasons: first, this is a retrospective study with certain limitations due to study design, such as a lack of randomization; second, we could not withhold an effective treatment from patients and include patients with diabetic macular edema in an untreated control group.

With the RetmarkerDR automated fundus analysis, additional insights could be presented on the effects of ranibizumab treatment. Not only an absolute reduction of the number of MAs could be shown, but also by dynamic analysis a higher decrease in new MAs was found. Those promising findings not only help to better understand the disease and dynamics, but might also serve as an additional quantification of the treatment response in the future. This might add to CRT as we move more and more towards response-driven treatment schemes.

\section{Acknowledgment}

The study was supported by a grant by Novartis.

\section{Disclosure Statement}

Financial interest: none.

\section{References}

1 Harris MI: Diabetes in America: epidemiology and scope of the problem. Diabetes Care 1998;21(suppl 3):C11-C14.

2 Claessen H, Genz J, Bertram B, Trautner C, Giani G, Zöllner I, et al: Evidence for a considerable decrease in total and cause-specific incidences of blindness in Germany. Eur J Epidemiol 2012;27:519-524.

3 Wormald R, Henshaw K, Smeeth L: Evidence-Based Ophthalmology. London, BMJ Books, 2008.

4 Photocoagulation for diabetic macular edema. Early Treatment Diabetic Retinopathy Study report number 1. Early Treatment Diabetic Retinopathy Study research group. Arch Ophthalmol 1985;103:17961806.

5 Haritoglou C, Kook D, Neubauer A, Wolf A, Priglinger S, Strauss R, et al: Intravitreal beva- cizumab (Avastin) therapy for persistent diffuse diabetic macular edema. Retina 2006;26: 999-1005.

-6 Mitchell P, Bandello F, Schmidt-Erfurth U, Lang GE, Massin P, Schlingemann RO, et al: The RESTORE study: ranibizumab monotherapy or combined with laser versus laser monotherapy for diabetic macular edema. Ophthalmology 2011;118:615625.

7 Do DV, Nguyen QD, Boyer D, SchmidtErfurth U, Brown DM, Vitti R, et al: Oneyear outcomes of the Da Vinci Study of VEGF Trap-Eye in eyes with diabetic macular edema. Ophthalmology 2012;119:16581665.

8 Cunha-Vaz J: Characterization and relevance of different diabetic retinopathy phenotypes. Dev Ophthalmol 2007;39:13-30.
-9 Nunes S, Pires I, Rosa A, Duarte L, Bernardes $\mathrm{R}$, Cunha-Vaz JE: Microaneurysm turnover is a biomarker for diabetic retinopathy progression to clinically significant macular edema: findings for type 2 diabetics with nonproliferative retinopathy. Ophthalmologica 2009; 223:292-297.

10 Haritoglou C, Kernt M, Neubauer A, Gerss J, Oliveira CM, Kampik A, et al: Microaneurysm formation rate as a predictive marker for progression to clinically significant macular edema in nonproliferative diabetic retinopathy. Retina 2014;34:157-164.

11 Ribeiro ML, Nunes SG, Cunha-Vaz JG: Microaneurysm turnover at the macula predicts risk of development of clinically significant macular edema in persons with mild nonproliferative diabetic retinopathy. Diabetes Care 2012;36:1254-1259. 
12 Kohner EM, Sleightholm M: Does microaneurysm count reflect severity of early diabetic retinopathy? Ophthalmology 1986;93:586589.

13 Reznicek L, Kernt M, Haritoglou C, Ulbig M, Kampik A, Neubauer AS: Correlation of leaking microaneurysms with retinal thickening in diabetic retinopathy. Int J Ophthalmol 2011;4:269-271.

14 Vincze P, Madácsy L, Petheö I, Brooser G: Prognostic significance of retinal microaneurysm number and localization in type-1 diabetes mellitus (in Hungarian). Orv Hetil 2001; 142:2015-2020.
15 Sjølie AK, Klein R, Porta M, Orchard T, Fuller J, Parving $\mathrm{HH}$, et al: Retinal microaneurysm count predicts progression and regression of diabetic retinopathy. Post-hoc results from the DIRECT Programme. Diabet Med 2011;28:345-351.

16 Hellstedt T, Immonen I: Disappearance and formation rates of microaneurysms in early diabetic retinopathy. Br J Ophthalmol 1996; 80:135-139.

17 Klein R, Meuer SM, Moss SE, Klein BE: Retinal microaneurysm counts and 10-year progression of diabetic retinopathy. Arch Ophthalmol 1995;113:1386-1391.
8 Lee SN, Chhablani J, Chan CK, Wang H, Barteselli G, El-Emam S, et al: Characterization of microaneurysm closure after focal laser photocoagulation in diabetic macular edema. Am J Ophthalmol 2013;155:905-912.e2.

19 Sachdev N, Gupta V, Abhiramamurthy V, Singh R, Gupta A: Correlation between microaneurysm closure rate and reduction in macular thickness following laser photocoagulation of diabetic macular edema. Eye 2007;22:975-977.

20 Kernt M, Cserhati S, Seidensticker F, Liegl R, Kampik A, Neubauer A, et al: Improvement of diabetic retinopathy with intravitreal ranibizumab. Diabetes Res Clin Pract 2013;100:e11e13. 